\title{
Analysis of noise temperature sensitivity for the design of a broadband thermal noise primary standard
}

\author{
Alejandro Díaz-Morcillo , Antonio Lozano-Guerrero , \\ Jaime Fornet-Ruiz' and Juan Monzó-Cabrera
}

\begin{abstract}
A broadband primary standard for thermal noise measurements is presented and its thermal and electromagnetic behaviour is analysed by means of a novel hybrid analytical-numerical simulation methodology. The standard consists of a broadband termination connected to a $3.5 \mathrm{~mm}$ coaxial airline partially immersed in liquid nitrogen and is designed in order to obtain a low reflectivity and a low uncertainty in the noise temperature. A detailed sensitivity analysis is made in order to highlight the critical characteristics that mostly affect the uncertainty in the noise temperature, and also to determine the manufacturing and operation tolerances for a proper performance in the range $10 \mathrm{MHz}$ to $26.5 \mathrm{GHz}$. Aspects such as the thermal bead design, the level of liquid nitrogen or the uncertainties associated with the temperatures, the physical properties of the materials in the standard and the simulation techniques are discussed.
\end{abstract}

\section{Introduction}

Primary noise standards are of key importance for noise measurements in the radiofrequency and microwave range. They allow the calibration of secondary standards, noise sources and noise measurement equipment, thus providing traceability to the whole measurement chain.

National Metrology Institutes have developed different primary standards. Some examples are the French standards (LNE) based on a set of waveguides ranging from $8.2 \mathrm{GHz}$ to $40 \mathrm{GHz}$ [1] or the German standards (PTB), both in waveguide (X-band, $12.4 \mathrm{GHz}$ to $18 \mathrm{GHz}$ ) [2] and in coaxial technology $(0.1 \mathrm{GHz}$ to $10 \mathrm{GHz})[3]$. These and other noise standards (NIST, NPL) are compared in [4]. Other national standards are currently in progress [5].

The National Institute for Aerospace Technology (INTA, Spain) is currently developing a broadband $(10 \mathrm{MHz}$ to $26.5 \mathrm{GHz}$ ) primary thermal noise standard based on coaxial technology, which will constitute the future Spanish national standard. This paper describes the design of the different components of this standard in order to minimize the overall uncertainty. With this goal in mind, a complete characterization of the noise temperature and its uncertainty, based on analytical formulations and numerical methods, is carried out.

The coaxial technology has been chosen in order to obtain a broadband behaviour. The reference for the standard design has been the NIST coaxial thermal noise primary standard described in [6], based on a $7 \mathrm{~mm}$ coaxial airline + termination submerged in liquid nitrogen, and working in the range $1 \mathrm{GHz}$ to $12.4 \mathrm{GHz}$. In fact, the analytical approach here is strongly related to that described in [6]. The necessity of increasing the bandwidth up to the range $10 \mathrm{MHz}$ to $26.5 \mathrm{GHz}$ requires the use of a $3.5 \mathrm{~mm}$ coaxial airline and a new design of different parts of the standard, in order to keep small values of noise temperature and reflectivity at the input port and, what is more important, accurate and stable values for these magnitudes.

In sections 2 to 6 this primary standard, with its different parts, and the computational methodology for obtaining its noise temperature are presented. Next, in sections 7 and 8 , the influence of the different elements/characteristics of the 


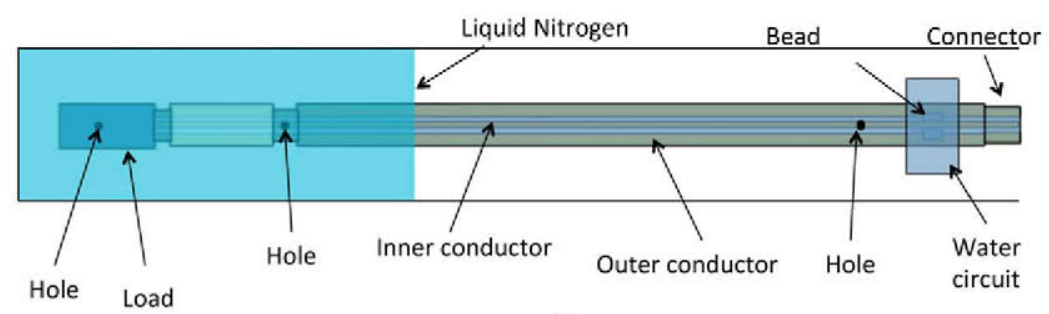

(a)

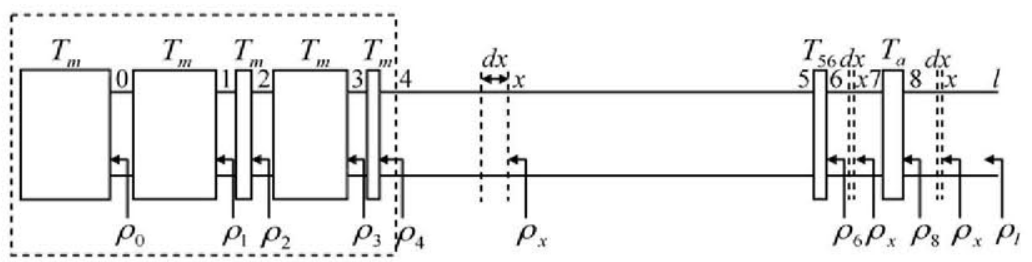

(b)

Figure 1. Thermal noise primary standard: $(a)$ sketch and $(b)$ computational scheme.

standard (termination, airline, thermal bead, level of liquid nitrogen, etc) on the reflection coefficient and on the noise temperature is analysed in a rigorous way in order to determine the sensitivity to the different uncertainty sources, and with the aim of prescribing manufacturing and operation characteristics and tolerances for reducing the uncertainty.

\section{New thermal noise primary standard}

The standard, depicted in figure $1(a)$, consists of a broadband coaxial termination submerged in liquid nitrogen and connected to a $3.5 \mathrm{~mm}$ airline partially submerged in liquid nitrogen, which allows the temperature gradient from the boiling point of liquid nitrogen $\left(T_{\mathrm{m}}\right)$ to the room temperature $\left(T_{\mathrm{a}}\right)$. Moreover, both the termination and the airline outer conductor are drilled in order to allow the liquid nitrogen to flow into the termination and part of the airline, and also to allow gaseous nitrogen to exit the airline in its upper part.

A water circuit around the airline and a thermal bead between the airline conductors are necessary in order to force the room temperature in both the inner and outer conductors at the upper edge of the line. Since the liquid nitrogen is kept in contact with both conductors in the lower zone of the line, the bead is only necessary in the upper zone.

This configuration provides important characteristics to the standard, such as

- Nearly constant temperature distribution at the termination.

- Nearly constant temperature distribution at the input port of the standard.

- Known temperature distribution throughout the airline.

\section{Computation of the noise temperature of the standard}

The noise standard can be analysed as a termination connected to a cascade of two-port networks and lines, as shown in figure $1(b)$, where the parts of the standard with a constant temperature distribution can be treated as discrete networks. The noise temperature of the standard is the sum of the contributions of the termination and each element in the line to the noise at the test port $(l)$.

The noise temperature generated by the termination that reaches $l$ is

$$
T_{\mathrm{m}} \kappa_{0 l},
$$

where $\kappa_{0 l}$ is the ratio of the available power in $l$ to the available power in 0 , which is obtained as

$$
\kappa_{0 l}=\frac{\left(1-\left|\rho_{0}\right|^{2}\right)\left|S_{21}\right|_{0 l}^{2}}{\left.\left(1-\left|\rho_{l}\right|^{2}\right)\left|1-S_{11}\right|_{0 l} \rho_{0}\right|^{2}},
$$

where $\rho_{i}$ is the reflection coefficient towards the termination at point $i$, and $\left.S_{11}\right|_{i j}$ and $\left.S_{21}\right|_{i j}$ are the scattering parameters of the network from point $i$ to point $j$.

The noise temperature generated in $l$ by the element $i j$ at constant temperature $T_{i j}$ is obtained as

$$
T_{i j}\left(1-\kappa_{i j}\right) \kappa_{j l}
$$

where $\kappa_{i j}$ and $\kappa_{j l}$ follow (2).

The thermal noise contribution of the line parts with a temperature gradient is obtained by integrating the thermal noise generated at each $\mathrm{d} x$. Thus, for a piece of line extending from $i$ to $j$, the noise temperature is

$$
2 \int_{i}^{j} T_{x} \alpha_{x} \mathrm{e}^{-2 \int_{x}^{j} \alpha_{y} \mathrm{~d} y} \frac{\left(1+\left|\rho_{x}\right|^{2}\right)\left|S_{21}\right|_{j l}^{2}}{\left.\left(1-\left|\rho_{l}\right|^{2}\right)\left|1-S_{11}\right|_{j l} \rho_{j}\right|^{2}} \mathrm{~d} x,
$$

where $T_{x}$ and $\alpha_{x}$ are the temperature and the line attenuation constant at point $x$, respectively, and the reflection coefficient at point $x_{1}$ can be obtained from the reflection coefficient at a close point $x_{0}$ as

$$
\rho_{x_{1}}=\rho_{x_{0}} \mathrm{e}^{-2 \gamma_{x_{0}}\left(x_{1}-x_{0}\right)}
$$

where $\gamma_{x}$ is the line propagation constant at $x$, whose real part is the attenuation constant $\alpha_{x}$ and the imaginary part is the phase constant $\beta_{x}$. 
Likewise, the reflection coefficient after a two-port network can be calculated as

$$
\rho_{2}=s_{22}+\frac{s_{12} s_{21} \rho_{1}}{1-s_{11} \rho_{1}},
$$

where $\rho_{1}$ and $\rho_{2}$ are the reflection coefficients towards the termination at ports 1 and 2 of the network, and $s_{i j}$ are the scattering parameters of the network.

It is important to note that, although the reflection coefficient is not the reference magnitude of the standard, it is of key importance to keep it as low as possible, in order to reduce the noise temperature oscillations and also its associated uncertainty. Knowing the termination reflection coefficient and the different scattering matrices in figure $1(b)$ it is possible to obtain with (5) and (6) the reflection coefficient at any point of the standard, which is necessary for the noise temperature calculation, as we have seen above.

The scattering parameters of a discontinuity (for instance, the nitrogen phase change) can be obtained as

$$
\begin{gathered}
s_{11}=-s_{22}=\frac{Z_{02}-Z_{01}}{Z_{01}+Z_{02}} \\
s_{21}=s_{12}=\frac{2 \sqrt{Z_{01} Z_{02}}}{Z_{01}+Z_{02}}
\end{gathered}
$$

where $Z_{02}$ and $Z_{01}$ are the characteristic impedances at both sides of the discontinuity.

Nevertheless, in more complex parts of the standard, such as orifices and the thermal bead, a numerical simulation will be necessary for obtaining the scattering parameters.

The temperature distribution throughout the line can be obtained by means of a finite element simulation of the parabolic heat equation. In this work the Partial Differential Equation Toolbox in Matlab rotational symmetry allows a 2D numerical simulation.

In a first approximation, assuming low losses in the line, the attenuation constant in $x$ is the sum of the attenuation constant due to the conductor losses $\alpha_{c x}$, and the attenuation constant due to dielectric losses $\alpha_{\mathrm{d} x}$. This latter contribution should be only taken into account for the liquid nitrogen and for the thermal bead stretches. For them

$$
\alpha_{\mathrm{d} x}=\frac{\pi}{\lambda_{0}} \sqrt{\varepsilon_{x}^{\prime}} \tan \delta_{x}
$$

where $\lambda_{0}$ is the vacuum wavelength and $\varepsilon_{x}^{\prime}$ and $\tan \delta_{x}$ are the dielectric constant and the loss tangent, respectively, of the dielectric material between the inner and the outer conductors.

Assuming a homogeneous material for both conductors, the attenuation constant due to conductor losses at point $x$ can be obtained as

$$
\alpha_{\mathrm{c} x}=\frac{R_{s x} \sqrt{\varepsilon_{x}^{\prime}}}{240 \pi a} \frac{1+a / b}{\ln (a / b)},
$$

where $a$ and $b$ are the inner radius of the outer conductor and the radius of the inner conductor, respectively, and $R_{s x}$ is the conductor surface resistance, which depends on the frequency and, through the electrical conductivity, on the temperature:

$$
R_{s x}=\sqrt{\frac{\pi f \mu_{0}}{\sigma_{x}}} .
$$

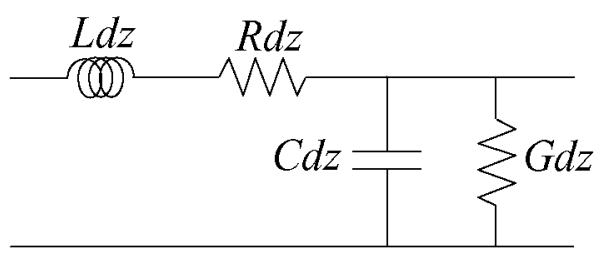

Figure 2. Circuital model of a transmission line of length $\mathrm{d} z$.

In (11) $\mu_{0}$ is the vacuum magnetic permeability, $f$ the frequency and $\sigma_{x}$ the electric conductivity in $x$.

But, in general, the inner and the outer conductors will have different temperatures and, therefore, it is necessary to split both contributions as

$$
\alpha_{c x}=\frac{\sqrt{\varepsilon_{x}^{\prime}}}{240 \pi \ln (a / b)}\left(\frac{R_{s x o}}{a}+\frac{R_{s x i}}{b}\right)
$$

where $R_{s x o}$ and $R_{s x i}$ are the surface resistance for the outer and the inner conductors in $x$, respectively.

The phase constant at point $x$ can be calculated, following the same low loss approximation, as

$$
\beta_{x}=2 \pi f \sqrt{\mu_{0} \varepsilon_{0} \varepsilon_{x}^{\prime}}
$$

where $\varepsilon_{0}$ is the vacuum electric permittivity.

The values of the characteristic impedance in the coaxial line at point $x$ are obtained by the approximation

$$
Z_{0 x}=\frac{60}{\sqrt{\varepsilon_{x}^{\prime}}} \ln \frac{a}{b} .
$$

An exact calculation of the characteristic impedance and the propagation constant can be performed by calculating the line parameters $R, L, G$ and $C$, corresponding to the line model of figure 2 , as

$$
\begin{gathered}
R=\frac{1}{2 \pi}\left(\frac{R_{s o}}{a}+\frac{R_{s i}}{b}\right) \\
L=\frac{\mu_{0}}{2 \pi} \ln \frac{a}{b} \\
G=\frac{2 \pi \omega \varepsilon_{0} \varepsilon^{\prime} \tan \delta}{\ln (a / b)} \\
C=\frac{2 \pi \varepsilon_{0} \varepsilon^{\prime}}{\ln (a / b)}
\end{gathered}
$$

where $\omega$ is the angular frequency, and applying

$$
\begin{gathered}
Z_{0}=\sqrt{\frac{R+\mathrm{j} \omega L}{G+\mathrm{j} \omega C}} \\
\gamma=\sqrt{(R+\mathrm{j} \omega L)(G+\mathrm{j} \omega C)} .
\end{gathered}
$$

Nevertheless, a comparison between the exact and the approximated values showed a very low relative error, which can be considered negligible.

One final remark in noise temperature calculation is the quantum effect. Equations (1), (3) and (4) are based on the approximate relationship between noise power and temperature

$$
P=k T B,
$$

where $k$ is the Boltzmann constant and $B$ the signal bandwidth. 


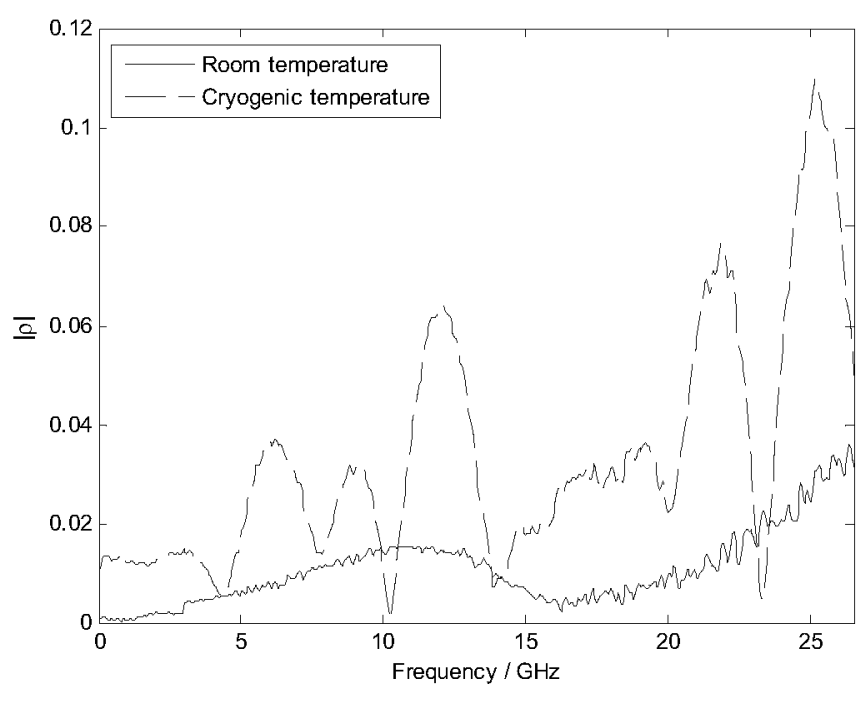

Figure 3. Magnitude of the reflection coefficient in the Agilent 909D termination for room temperature and $20 \mathrm{~min}$ after immersion in liquid nitrogen.

In order to avoid the systematic error produced by this approximation, which becomes more important at high frequencies, the temperature in equations (1), (3) and (4) must be the radiation temperature, related to the physical temperature by

$$
T_{\mathrm{r}}=\frac{h f / k}{\mathrm{e}^{h f / k T}-1},
$$

where $h$ is the Planck constant. For the upper frequency boundary $(26.5 \mathrm{GHz})$ the use of (22) avoids a systematic error of $+0.48 \mathrm{~K}$ in the noise temperature of the standard.

\section{Broadband coaxial termination}

After having analysed different terminations in search of a broadband low reflectivity behaviour, the Agilent 909D termination [7] was chosen for the primary standard.

Thermal simulations with the liquid nitrogen outside the termination yield thermal gradients greater than $5 \mathrm{~K}$ from the termination surface to the centre zone. Therefore, a modification in the termination, i.e. an orifice at the bottom of the termination, is needed in order to let the liquid nitrogen flow into it.

The reflection coefficient of the termination was measured with a vector network analyser (VNA). As expected, it showed an increase in reflectivity when the termination was submerged in liquid nitrogen, as shown in figure 3 . This measurement introduces an error associated with the VNA and its calibration. Moreover, this calibration is made at room temperature, whilst the termination measurement is made under cryogenic conditions. It is possible, nevertheless, to obtain an accurate cryogenic measurement by measuring the reflection coefficient of the noise standard and, since the line is completely modelled, de-embedding this measurement backwards to the termination plane. In fact, this noise standard could be used too as a VNA calibration tool for cryogenic measurements.

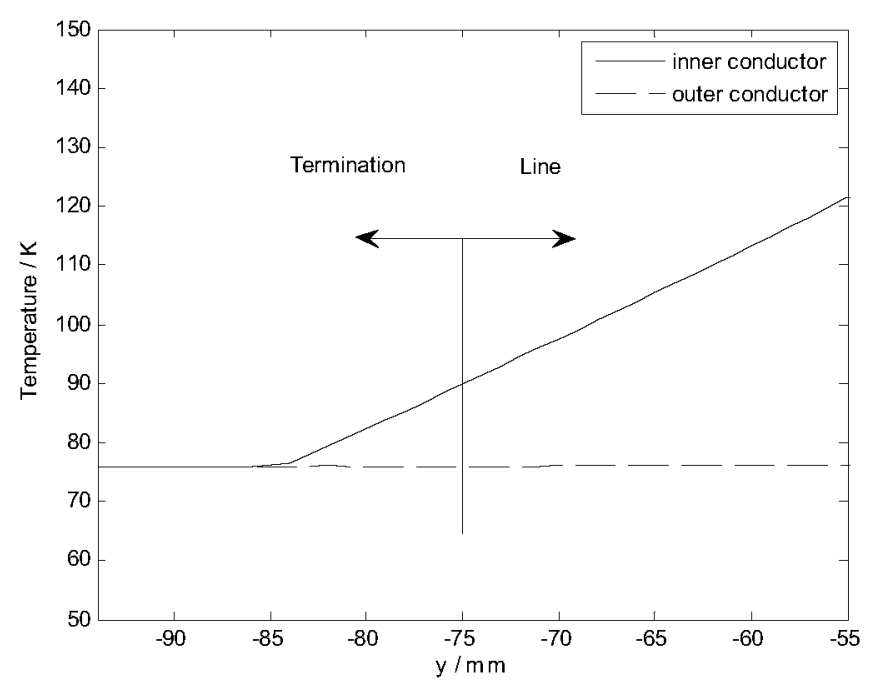

Figure 4. Temperature distribution in the termination and lower part of the line when liquid nitrogen is outside the line.

A thermal simulation of the termination connected to the transmission line shows (figure 4) a noticeable gradient in the upper part of the termination, when there is no liquid nitrogen inside the line. Therefore, in order to accomplish the requirement of constant distribution of temperature along the termination, the liquid nitrogen must be inside the transmission line too. Moreover, once the introduction of nitrogen inside the line has been decided, a new termination can be defined as the commercial termination plus the submerged segment of the transmission line, thus simplifying the scheme of the primary standard for the noise temperature calculation, as shown in figure $1(b)$.

\section{Transmission line}

A $15 \mathrm{~cm}$ gold-plated beryllium-copper (C17200) airline was chosen as the base for the transmission line in the standard. In order to allow the introduction of liquid nitrogen in the line, a lower orifice (for liquid nitrogen input) and an upper orifice (for gaseous nitrogen output) were created. Different drill sizes were numerically simulated by means of the finite element method in frequency domain (FEFD) and a tradeoff between acceptable volume of flow and low impact in the standard electromagnetic behaviour was found for a diameter value of $2 \mathrm{~mm}$. Figure 5 shows the $s_{11}$ and $s_{21}$ magnitudes obtained for this case.

Although more accurate results can be obtained with a constant level of liquid nitrogen, for the sake of practical use of the standard this level can vary, as in [6], between a lower and an upper bound. Obviously, the maximum level cannot be close to the thermal bead, since a minimum length of line is needed for a smooth temperature gradient that can accomplish an almost constant temperature distribution after the bead. In order to study the influence in the results section, in a first approach, we fix the lower level just over the lower orifice and the upper level $5 \mathrm{~cm}$ higher.

The gold plating of the line is an important characteristic, mainly at lower frequencies, where the skin depth increases 


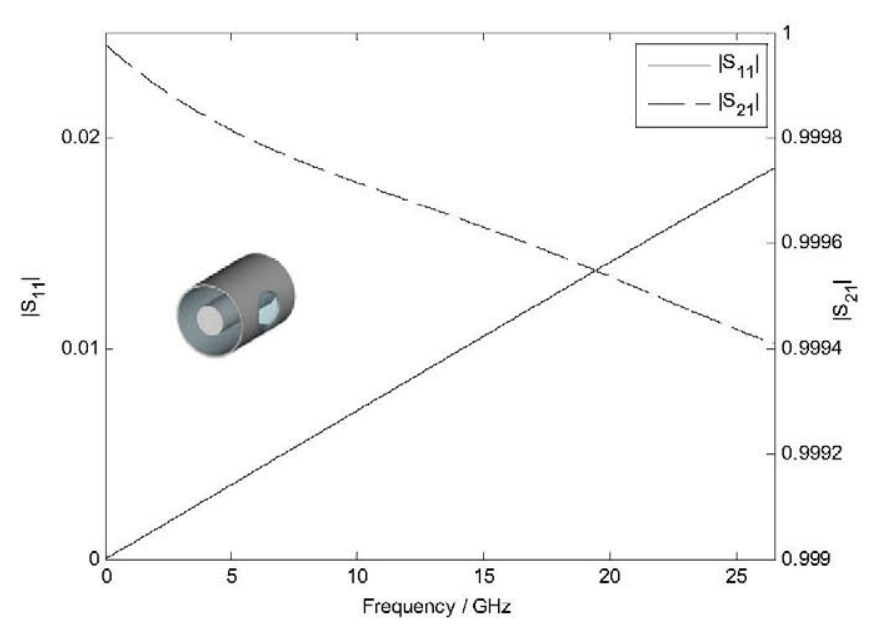

Figure 5. Scattering parameters magnitudes for a $2 \mathrm{~mm}$ orifice in the line.

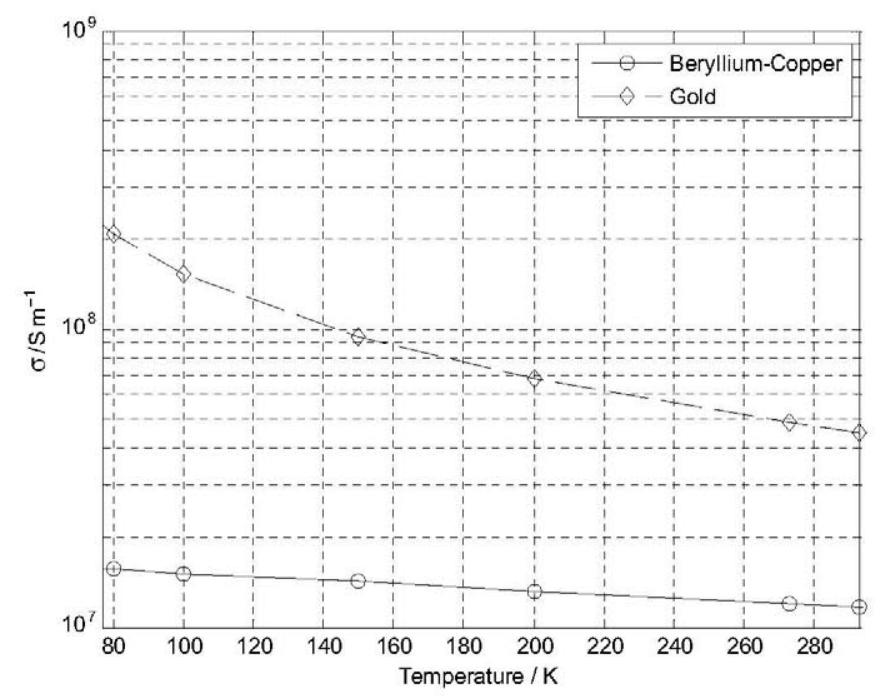

Figure 6. Variation of gold and beryllium-copper electrical conductivities with temperature.

and the assumption of infinite gold thickness no longer applies in the calculation of $\alpha_{\mathrm{cx}}$. In [6], where the lower operation frequency is $1 \mathrm{GHz}$, the effect of the finite gold thickness is characterized by an uncertainty in the resistivity, which is assumed for a homogeneous gold conductor. In our standard, where the lowest operation frequency is $10 \mathrm{MHz}$, a rather thick gold plating $(\sim 50 \mu \mathrm{m})$ would be needed to keep a small uncertainty at lower frequencies, thus increasing the manufacturing tolerances. Therefore, the effect of the base conductor (beryllium-copper) is taken into account in the calculation of $\alpha_{c x}$ following [8] and consequently, only the uncertainty contribution due to errors in the electrical conductivity specifications of the conductor materials has to be assessed. Figure 6 shows the dependence of the gold [9] and the beryllium-copper [10] electrical conductivities on temperature.

\section{Thermal bead}

The main goal of the thermal bead is to provide a good thermal contact between the inner and the outer conductors in order to establish at the upper part of the line the temperature forced by the water circuit, which stays in contact with the outer conductor. For that reason, the material of the thermal bead must exhibit a high thermal conductivity, but also a low electrical permittivity, since it is of key importance to make the bead as electromagnetically transparent as possible. Thus, a low dielectric constant will reduce the bead reflectivity and a low loss tangent will reduce its losses and, consequently, the noise generation. The beads chosen for the primary standard in [6] were made of beryllium oxide. Due to the high toxicity of this compound, an alternative material was sought. Different ceramics such as silicon carbide, aluminium nitride or boron nitride were found as potential candidates. Table 1 shows the thermal conductivity and dielectric characteristics for these materials at room temperature. Finally, boron nitride, with a good combination of both properties, was chosen as bead material.

Once the material has been chosen, the bead must be designed in order to minimize its electromagnetic impact on the standard, that is, to reduce its reflection coefficient as much as possible for the whole frequency range. In order to achieve a good thermal contact between the inner and outer conductors it is evident that the centre of the bead must be in contact with both conductors. In this case, in order to obtain a characteristic impedance of $50 \Omega$, according to equation (14), the diameter of the inner conductor must be reduced. Another reason for using boron nitride instead of beryllium oxide in the bead is the diameter of the inner conductor in the central part of the bead. For boron nitride a reduction in the inner conductor diameter up to $0.66 \mathrm{~mm}$ is needed, whilst the higher permittivity of the beryllium oxide requires an even thinner inner conductor $(0.42 \mathrm{~mm}$ diameter) in order to keep the $50 \Omega$ continuity.

Although the impedance continuity is achieved for the TEM mode, the geometric and material discontinuity excites higher order modes in the coaxial line, and for frequencies higher than $23.43 \mathrm{GHz}$ the $\mathrm{TE}_{11}$ mode propagates along the bead, resulting in high magnitudes of $s_{11}$ for the bead. Moreover, the presence of higher order evanescent modes in the discontinuities and the propagation of the $\mathrm{TE}_{11}$ mode makes it necessary to use numerical methods for the determination of the bead scattering parameters. As in the case for the orifice, the FEFD method has been used.

Adjacent transitions to the bead are needed in order to reduce the bead reflectivity. In [14] tapered designs, continuous and stepped, were presented. In this solution, the transitions are partially filled with boron nitride in order to keep the $50 \Omega$ continuity.

An alternative to this design is an approach based on capacitive compensation, which was used in [6] for a $7 \mathrm{~mm}$ airline.

Two beads with different lengths $(4 \mathrm{~mm}$ and $6 \mathrm{~mm}$ ) have been analysed in this work. For a $3.5 \mathrm{~mm}$ airline and boron nitride as dielectric material, assuming the permittivity provided by the manufacturer, a compensation step of $0.25 \mathrm{~mm}$ is needed to minimize $\left|s_{11}\right|$ in both $4 \mathrm{~mm}$ and $6 \mathrm{~mm}$ beads. Figure 7 shows the design for the $6 \mathrm{~mm}$ bead, and figure 8 the magnitude of the scattering parameters $s_{11}$ and $s_{21}$ for both beads. As compared with the tapered design [14] 
Table 1. Dielectric and thermal properties of bead materials.

\begin{tabular}{llll}
\hline Material & $\varepsilon^{\prime}$ & $\tan \delta$ & $k_{T} / \mathrm{W} \mathrm{m}^{-1} \mathrm{~K}^{-1}$ \\
\hline Beryllium oxide & $6.86(8.5 \mathrm{GHz})[11]$ & $3.0 \times 10^{-4}(8.5 \mathrm{GHz})[11]$ & $200[6]$ \\
Aluminium nitride & $8.5(8.5 \mathrm{GHz})[11]$ & $3.0 \times 10^{-3}(8.5 \mathrm{GHz})[11]$ & $200[12]$ \\
Silicon carbide & $9.7(10 \mathrm{GHz})[11]$ & $1.0 \times 10^{-3}(10 \mathrm{GHz})[11]$ & $270[13]$ \\
Boron nitride grade AX05 & $4.0(8.8 \mathrm{GHz})^{\mathrm{a}}$ & $1.2 \times 10^{-3}(8.8 \mathrm{GHz})^{\mathrm{a}}$ & $70^{\mathrm{a}}$ \\
\hline
\end{tabular}

${ }^{a}$ Data from the manufacturer.

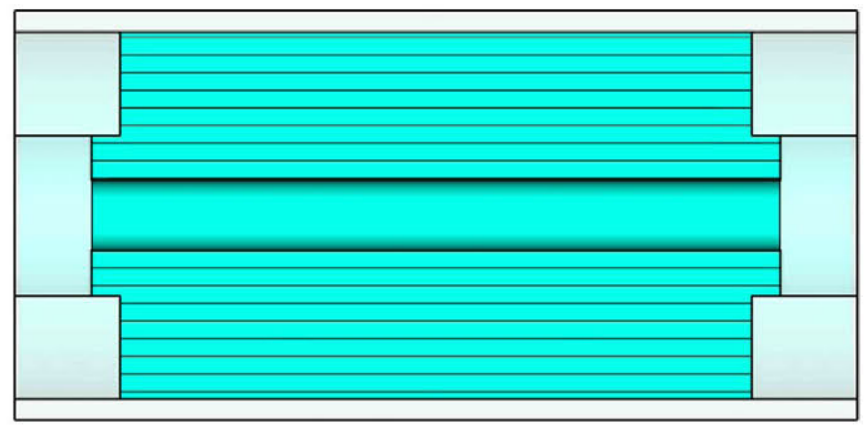

Figure 7. Thermal bead $(6 \mathrm{~mm})$ with capacitive compensation design.

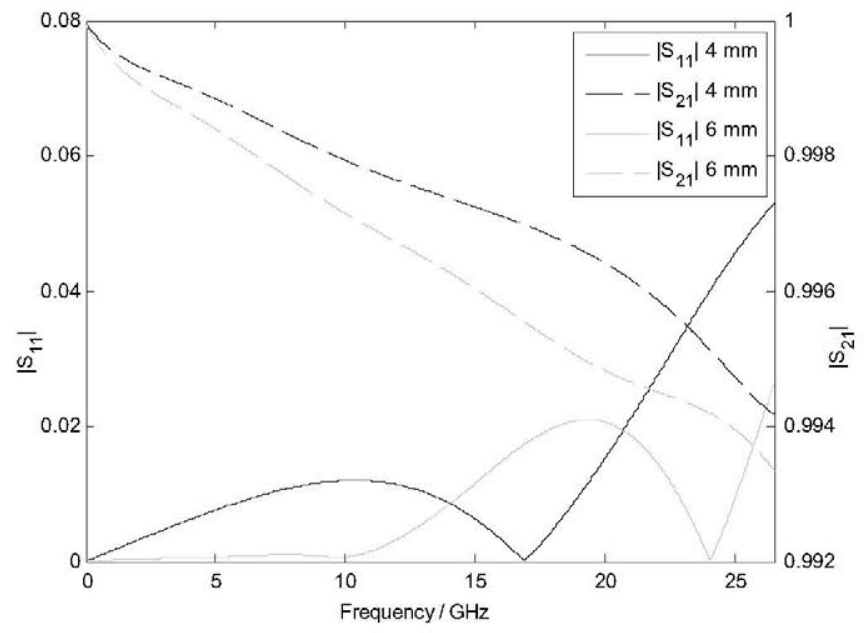

Figure 8. Magnitude of $s_{11}$ and $s_{21}$ of the thermal bead.

reflection decreases and transmission increases, as a result of the reduction in both the reflectivity and the losses, since the quantity of dielectric material is smaller. As a result of the above, this is finally the design chosen for the standard.

\section{Noise standard performance}

The complete knowledge of the standard geometry and the material properties allows us to calculate, by means of the formulation described in section 3 , the noise temperature at the input port of the standard. As mentioned in section 4, an accurate measurement of the reflection coefficient of the termination can only be obtained by de-embedding the measured reflection coefficient at the standard input port. For the analysis of the standard and without loss of generality, we assume that the submerged modified termination has the reflection coefficient of the termination at room temperature.
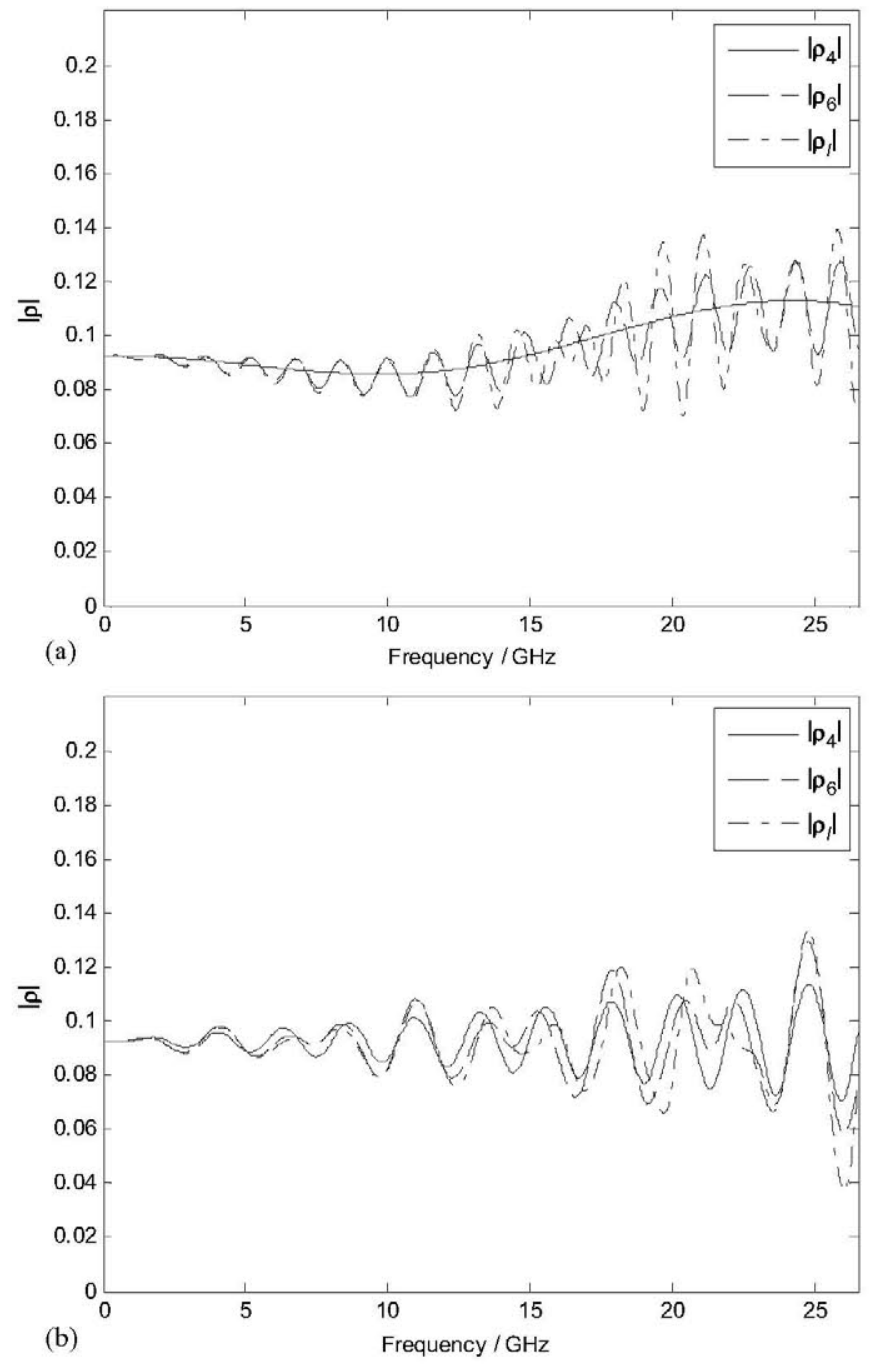

Figure 9. Magnitude of reflection coefficient throughout the standard (6 mm bead): $(a)$ lower level and $(b)$ upper level.

In fact, this value is masked by the higher value produced by the discontinuity at the nitrogen phase change.

In order to assess the contribution of the different parts of the standard, the reflection coefficient and the contribution to the noise temperature for a given level of liquid nitrogen can be calculated following the formulation in section 3. Figure 9 shows the reflection coefficient produced at different points of the standard for a nitrogen level in the lower bound and for a $6 \mathrm{~mm}$ long bead. The fluctuations in the reflection coefficient increase, reaching maximum values of 0.14 at the standard port for high frequencies. These internal reflections produce variations in the noise temperature as shown in figures 10 
and 11. These figures show the contributions to the noise temperature at the input port caused by the termination and by the different parts along the standard, respectively, when the $6 \mathrm{~mm}$ long bead is used. In this case, excluding the termination, the thermal bead produces the main contribution, due to its dielectric losses. Nevertheless, the contribution of the line with the temperature gradient (stretch 4-5) becomes important when the level of liquid nitrogen decreases. This is clearly observed in figure 12 , where the noise temperature of the standard increases when the nitrogen level decreases.

A constant temperature (the average temperature) is assumed in the numerical simulations for the upper orifice and for the thermal bead. Moreover, the temperature distribution at the upper port should be constant. Therefore, there is an upper limit for the nitrogen level where:

- The assumption of constant temperature distribution at the thermal bead or at the upper orifice can no longer be considered a good approximation.

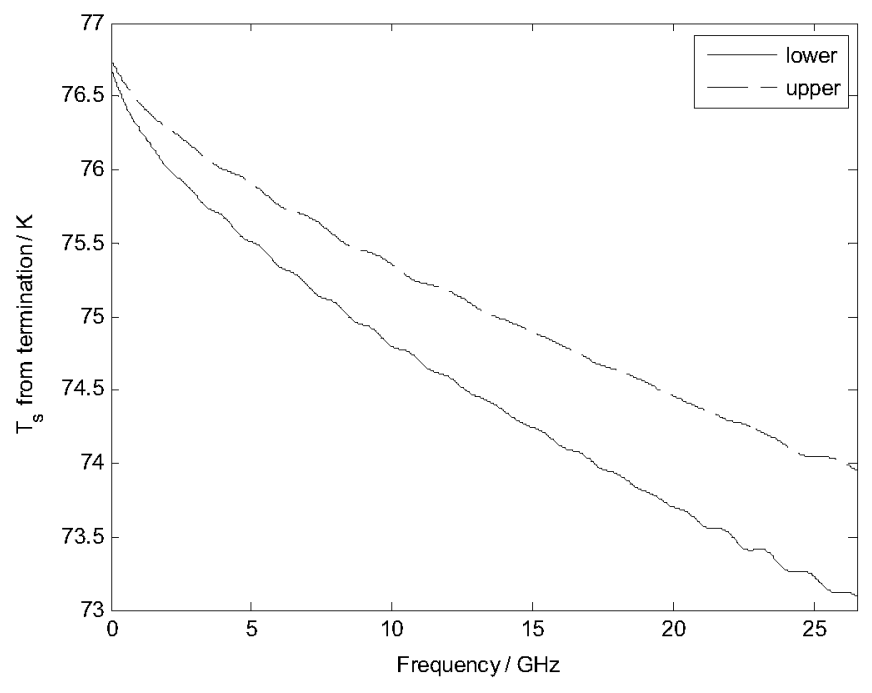

Figure 10. Termination contribution to noise temperature $(6 \mathrm{~mm}$ bead).
- The inner and outer conductor temperatures at the standard port are too different.

Simulations of these parts for the edge temperatures constitute a measure of the uncertainty in the simulation, considering the uncertainty associated with the numerical method negligible. Figure 13 shows the temperature distribution in the upper part of the standard for two different beads ( $4 \mathrm{~mm}$ and $6 \mathrm{~mm}$ ) and the minimum and maximum $(+5 \mathrm{~cm})$ levels of liquid nitrogen. Obviously, the maximum level is the most limiting case, since the distance between the cryogenic and the room temperature is shorter. Temperature gradients in the $4 \mathrm{~mm}$ thermal bead of $7.5 \mathrm{~K}$ and a difference of $1.9 \mathrm{~K}$ between inner and outer conductor temperatures at the input port are obtained. If a $6 \mathrm{~mm}$ bead is used, the bead gradient is slightly increased up to $8.2 \mathrm{~K}$, but the temperature difference at the port is reduced to $1 \mathrm{~K}$. Considering the edge temperatures, in a very conservative approach, the effect of the temperature gradient in the bead leads to a variation in the temperature noise, which is shown in figure 14 for both beads and for the lower and upper nitrogen levels. The $6 \mathrm{~mm}$ bead increases the uncertainty associated with the temperature gradient, since the bead is longer, but, on the other hand, it decreases the temperature difference at the standard input port. Therefore, increasing the bead length improves the temperature uniformity at the standard port, although this length is limited by the uncertainty associated with the temperature gradient, and also by the uncertainty associated with the dielectric loss of the bead, as will be shown later, so the bead length cannot be indefinitely increased. Increasing the upper limit for the nitrogen level will increase both the uncertainty associated with the temperature distribution along the bead and the temperature differences at the port. Therefore, the upper limit is set to $+5 \mathrm{~cm}$ and the $6 \mathrm{~mm}$ bead is chosen as a trade-off between temperature distribution in the bead, temperature distribution at the port and required time for continuous operation of the standard. For more accurate results, the upper bound for the nitrogen level should be decreased and, consequently, the operation time would be reduced.

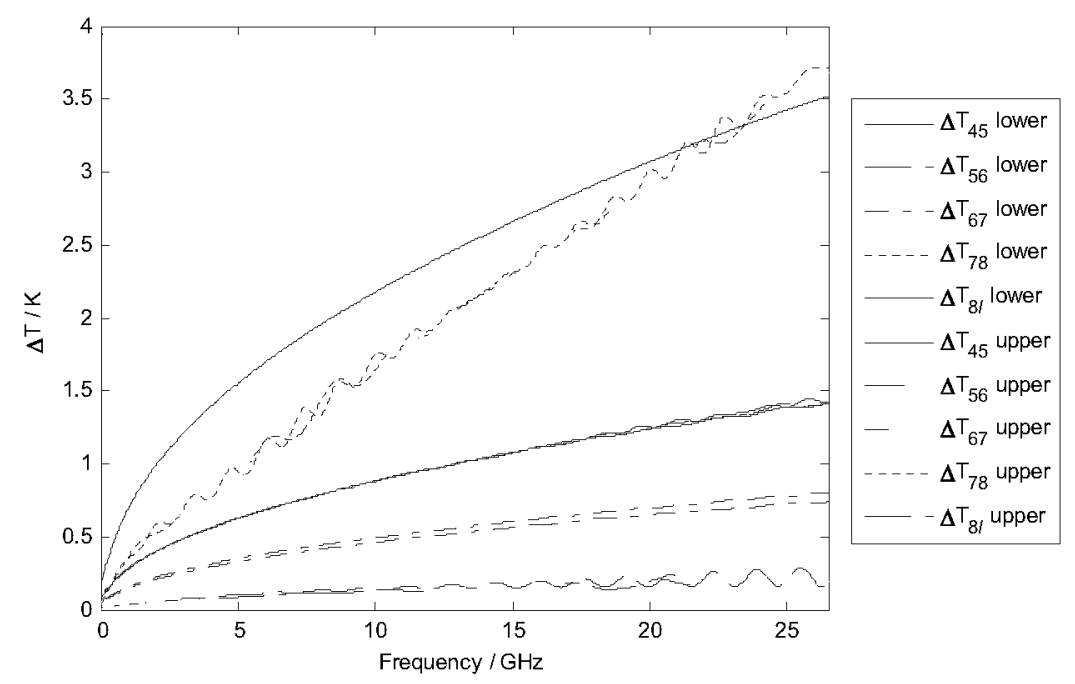

Figure 11. Transmission line contributions to noise temperature. 


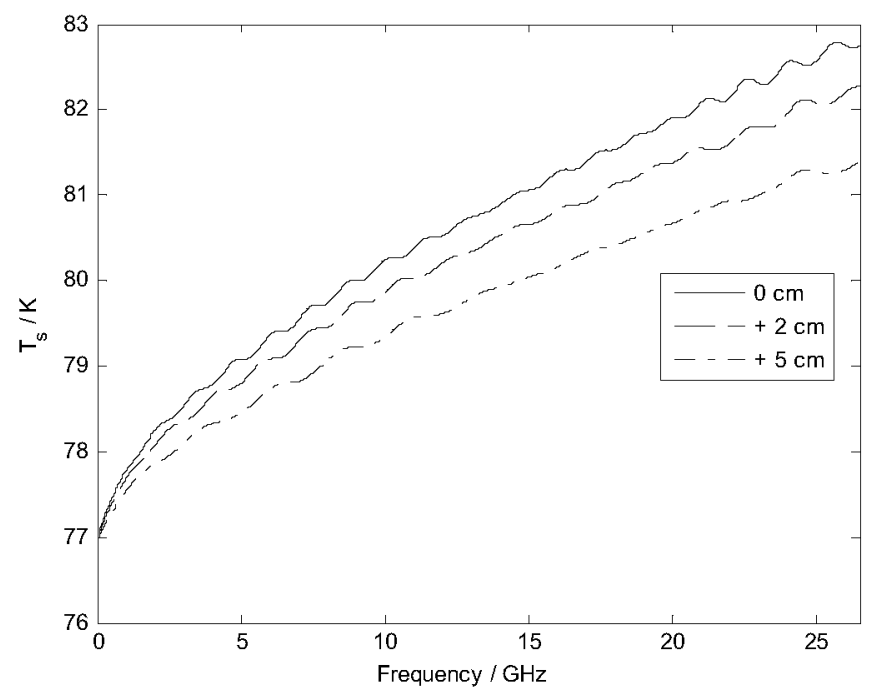

Figure 12. Noise temperature of the standard for different liquid nitrogen levels.

This study can be repeated for the hole. In this case, the uncertainty associated with the temperature distribution in the hole is much lower since the hole is shorter compared with the bead and it contains a lossless dielectric (gaseous nitrogen). The variation obtained in this case for $26.5 \mathrm{GHz}$ and for the $6 \mathrm{~mm}$ bead is lower than $\pm 0.003 \mathrm{~K}$ for the lower level and lower than $\pm 0.006 \mathrm{~K}$ for the upper one.

The uncertainty associated with the temperature gradient in the bead can be reduced by treating the bead as two lumped networks containing the edge bead modifications at constant temperatures, where the noise temperature is obtained by (3), and a transmission line between them with a known temperature distribution, where the noise temperature is obtained by (2). With this approach, the temperature distribution to take into account in the uncertainty computation lies around $1 \mathrm{~mm}$ or less and, therefore, its contribution to the overall uncertainty budget is much lower. The drawback of this approach is that (2) can only be used for a mono-mode transmission line, and the bead begins to propagate the second mode at the upper frequency range $(f>23.43 \mathrm{GHz})$, just where most of the uncertainty contributions reach their highest values.

All previous results were obtained for a $0.75 \mu \mathrm{m}$ thick gold plating. Taking into account that the beryllium-copper losses are included in the calculation of the conductor attenuation constant, it is not necessary to increase the gold plating thickness, which for values higher than $5 \mu \mathrm{m}$ can produce an increase in the manufacturing tolerances.

\section{Uncertainties}

The complexity of the noise temperature calculation, which involves thermal and electromagnetic numerical simulations along with analytical expressions (1)-(14) and (22), hinders the implementation of the GUM approach [15] for the uncertainty assessment. As a first step, in order to point out the key manufacturing and operation characteristics,
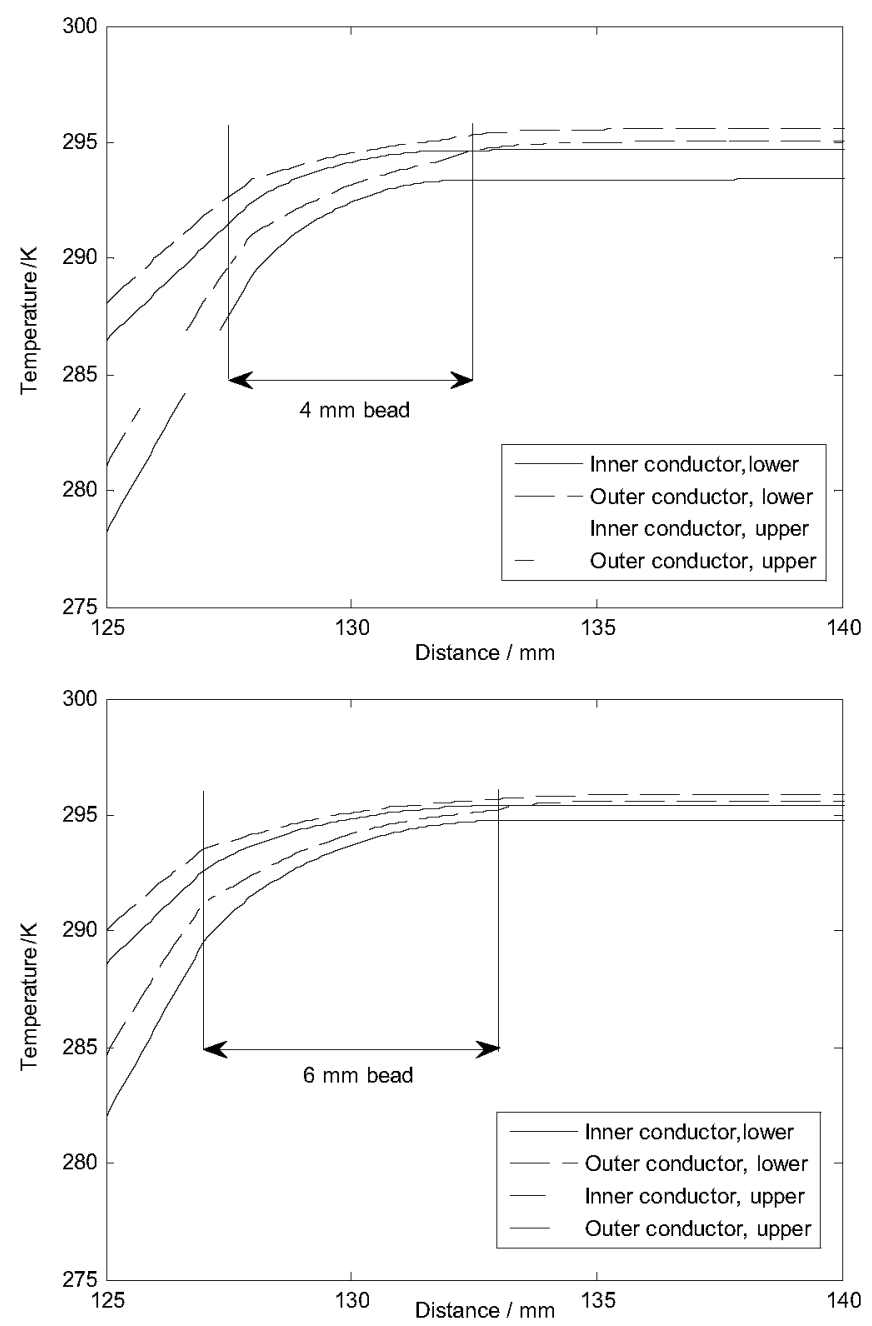

Figure 13. Temperature distribution in the upper part of the standard.

the influence of different parameter uncertainties on the variation of the standard noise temperature is analysed in this section.

Following in part the analysis contained in [6], several contributions are taken into account in order to identify the most critical parameters in the manufacturing and operation of the standard. For all the cases, the $6 \mathrm{~mm}$ thermal bead is used and the properties in table 2 are employed for the materials in the standard. Table 3 summarizes the results of this study, indicating the largest variations of the noise temperature throughout the broad frequency range for the lower and upper levels of liquid nitrogen.

\subsection{Temperature uncertainties}

The uncertainty in the water circuit temperature $T_{\mathrm{a}}$, due to variations in water temperature within the stabilization circuit, is quantified as $\pm 0.5 \mathrm{~K}$. The variation in the noise temperature produced by this uncertainty is relatively small, as shown in figure 15 .

The termination temperature $T_{\mathrm{m}}$ is assumed to be constant along that part of the standard submerged in liquid nitrogen. 


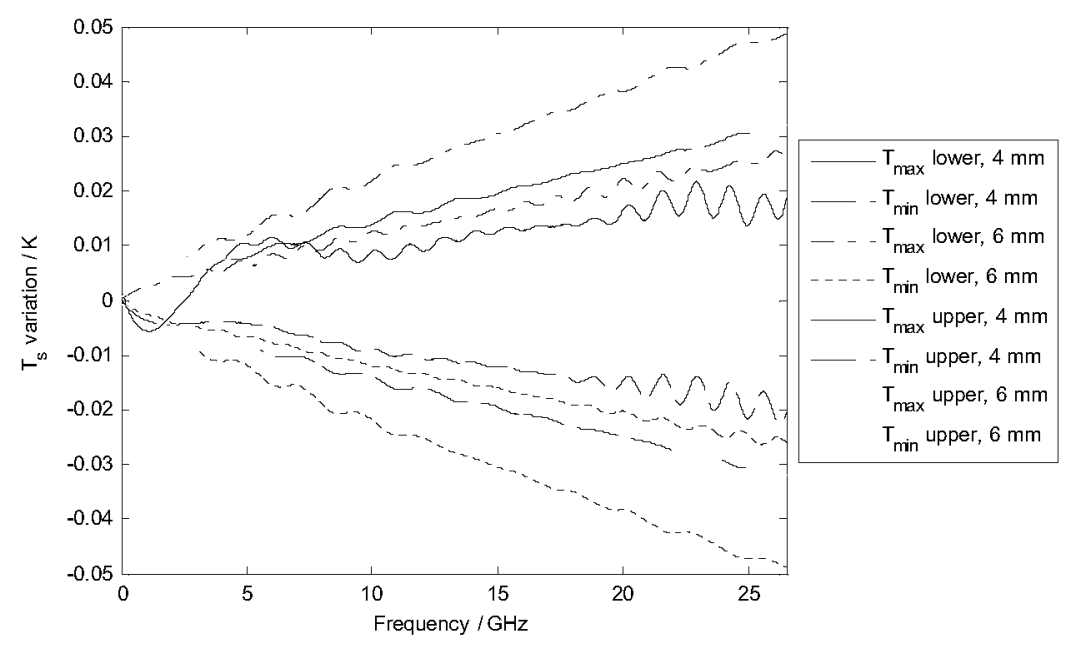

Figure 14. Noise temperature variation due to temperature gradient in the thermal bead.

Table 2. Properties of materials.

\begin{tabular}{lllllll}
\hline Material & $k_{T} / \mathrm{W} \mathrm{m}^{-1} \mathrm{~K}^{-1}$ & $c_{p} / \mathrm{J} \mathrm{kg}^{-1} \mathrm{~K}^{-1}$ & $\rho / \mathrm{kg} \mathrm{m}^{-3}$ & $\sigma / \mathrm{S} \mathrm{m}^{-1}$ & $\varepsilon^{\prime}$ & $\tan \delta$ \\
\hline Gold & - & - & - & figure 6 & - & - \\
Beryllium-copper & $35^{\mathrm{a}} / 105^{\mathrm{b}}$ & 376 & 8250 & figure 6 & - & - \\
Boron nitride & 70 & 350 & 1850 & - & 4.0 & 0.0012 \\
Liquid nitrogen & - & - & - & - & 1.45 & 0.00005 \\
Gaseous nitrogen & 0.0025 & 1040 & 1.2 & - & 1.00058 & 0 \\
Air & 0.025 & 1005 & 1.2 & - & 1.00059 & 0 \\
\hline
\end{tabular}

${ }^{a}$ At $77 \mathrm{~K}$.

${ }^{\mathrm{b}}$ At $297 \mathrm{~K}$.

Table 3. Sensitivity of noise temperature.

\begin{tabular}{llll}
\hline Uncertainty source & Source variation & $\begin{array}{l}\text { Largest } T_{\mathrm{s}} \text { variation } \\
\text { (lower level) }\end{array}$ & $\begin{array}{l}\text { Largest } T_{\mathrm{s}} \text { variation } \\
\text { (upper level) }\end{array}$ \\
\hline Water circuit temperature & $\pm 0.5 \mathrm{~K}$ & $\pm 0.014 \mathrm{~K}$ & $\pm 0.018 \mathrm{~K}$ \\
Hydrostatic pressure & - & $\pm 0.012 \mathrm{~K}$ & $\pm 0.03 \mathrm{~K}$ \\
Temp. gradient in bead & - & $\pm 0.028 \mathrm{~K}$ & $\pm 0.049 \mathrm{~K}$ \\
Temp. gradient in orifice & - & $\pm 0.003 \mathrm{~K}$ & $\pm 0.006 \mathrm{~K}$ \\
Liquid nitrogen level & $\pm 0.5 \mathrm{~mm}$ & $\pm 0.07 \mathrm{~K}$ & $\pm 0.07 \mathrm{~K}$ \\
Bead manufact. (diameter) & $\pm 0.01 \mathrm{~mm}$ & $+0.13 /-0.08 \mathrm{~K}$ & $+0.12 /-0.07 \mathrm{~K}$ \\
Bead manufact. (length) & $\pm 0.01 \mathrm{~mm}$ & $+0.019 /-0.022 \mathrm{~K}$ & $+0.013 /-0.014 \mathrm{~K}$ \\
Orifice manufact. (diameter) & $\pm 0.01 \mathrm{~mm}$ & $+0.028 /-0.078 \mathrm{~K}$ & $+0.012 /-0.068 \mathrm{~K}$ \\
Conductor roughness & $+2 \%$ & $+0.072 \mathrm{~K}$ & $+0.047 \mathrm{~K}$ \\
Dielectric constant & $\pm 5 \%$ & $+0.035 /-0.06 \mathrm{~K}$ & $\pm 0.04 \mathrm{~K}$ \\
Loss tangent & $\pm 5 \%$ & $+0.082 /-0.088 \mathrm{~K}$ & $+0.09 /-0.083 \mathrm{~K}$ \\
Electrical conductivity & $\pm 5 \%$ & $+0.12 /-0.1 \mathrm{~K}$ & $\pm 0.06 \mathrm{~K}$ \\
Thermal conductivity & $\pm 20 \%$ & negligible & negligible \\
Thermal simulation & - & $-0.0017 /-0.0027 \mathrm{~K}$ & $-0.0019 /-0.0034 \mathrm{~K}$ \\
Electromagnetic simulation & - & $+0.004 /-0.0005 \mathrm{~K}$ & $+0.004 /-0.0005 \mathrm{~K}$ \\
Numerical integration & - & $\pm 0.012 \mathrm{~K}$ & $\pm 0.01 \mathrm{~K}$ \\
\hline
\end{tabular}

The thermal simulations indicate a negligible temperature gradient $<10^{-3} \mathrm{~K}$ for this part of the standard. The uncertainty in the atmospheric and hydrostatic pressures can also be assessed. The atmospheric pressure at INTA's premises can be measured with an associated uncertainty of $\pm 10 \mathrm{~Pa}$, which produces, through the nitrogen pressure-temperature equation, a negligible temperature uncertainty of $0.0009 \mathrm{~K}$. We assume that the value for the atmospheric pressure is $95 \mathrm{kPa}$, which yields $76.8043 \mathrm{~K}$ as the termination temperature. Since the termination is distributed in the commercial termination and part of the airline, the hydrostatic pressure varies along its length. Therefore, this pressure is $0 \mathrm{kPa}$ in the nitrogen phase change level, and is given by $9.81 \times 0.808 \times 10^{3} d$ at the bottom of the termination, where $d$ is the height of the liquid nitrogen column. This hydrostatic pressure in the termination bottom is 301.2 Pa for the lower level and 697.53 Pa for the upper level. Thus, the average pressure in the termination is $95.151 \mathrm{kPa}$ for the lower boundary and $95.349 \mathrm{kPa}$ for the upper boundary, which increases the termination temperature up to $76.8175 \mathrm{~K}$ and $76.8349 \mathrm{~K}$, respectively. For these values, the uncertainty 


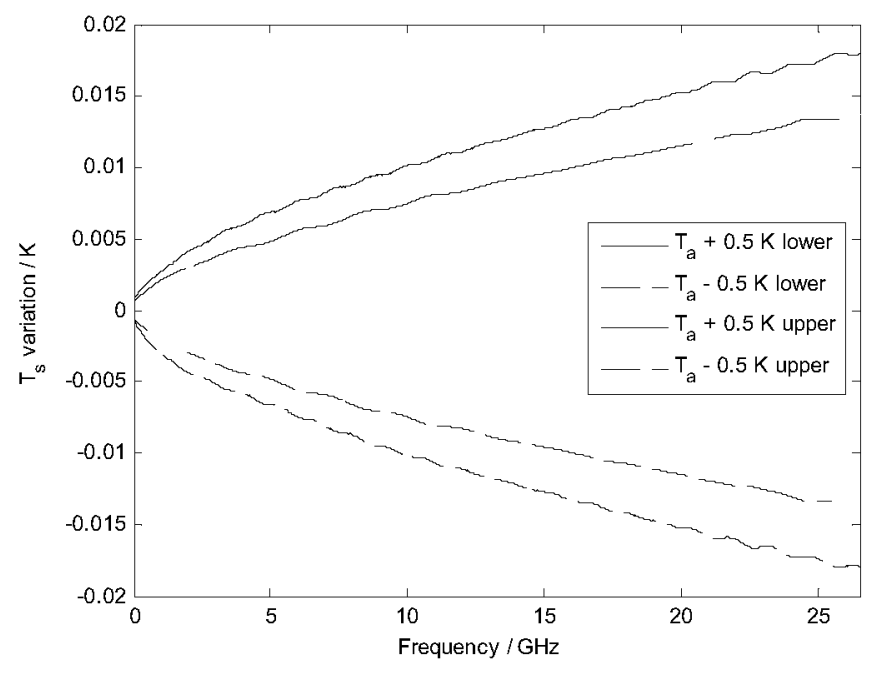

Figure 15. Noise temperature variation with the uncertainty of $T_{\mathrm{a}}$.

is $\pm 0.013 \mathrm{~K}$ and $\pm 0.031 \mathrm{~K}$, respectively. Following (1), this uncertainty is almost the uncertainty associated with the contribution of the termination to the noise temperature since $\kappa_{0 l}$ is close to 1 .

It would be possible to reduce the uncertainty associated with the pressure, mainly for intermediate or high levels of liquid nitrogen, by treating the submerged line as a distributed element, not as a part of the termination, with a temperature distribution depending on the pressure. However, this approach does not yield a significant reduction in the overall noise temperature uncertainty.

The temperature gradient in the thermal bead and in the upper orifice, where the temperature is assumed to be constant, is also an uncertainty source. This effect has been analysed in section 7, and the influence of the thermal bead on the noise temperature is shown in figure 14, whilst the influence of the orifice is considered negligible. The noise temperature variation can be reduced by decreasing the upper bound for the level of liquid nitrogen, thus reducing also the required time for operation with the standard.

\subsection{Nitrogen level}

The uncertainty associated with the level of the liquid nitrogen influences the temperature distribution and also the reflection coefficient along the standard. This second effect is the most important for the noise temperature uncertainty since it produces a displacement of the variations due to the internal reflections. The cryostat must therefore provide a system for measuring the nitrogen level with a low uncertainty. For instance, an uncertainty of $\pm 1 \mathrm{~mm}$ yields the variations shown in figure 16. In order to reduce such variations, an uncertainty of $\pm 0.5 \mathrm{~mm}$ in the measurement of the nitrogen level is advisable.

\subsection{Manufacturing tolerances}

The tolerances in the manufacturing of the thermal bead and the inner conductor modification are defined in the diameters and lengths of the different cylinders in the thermal bed, which

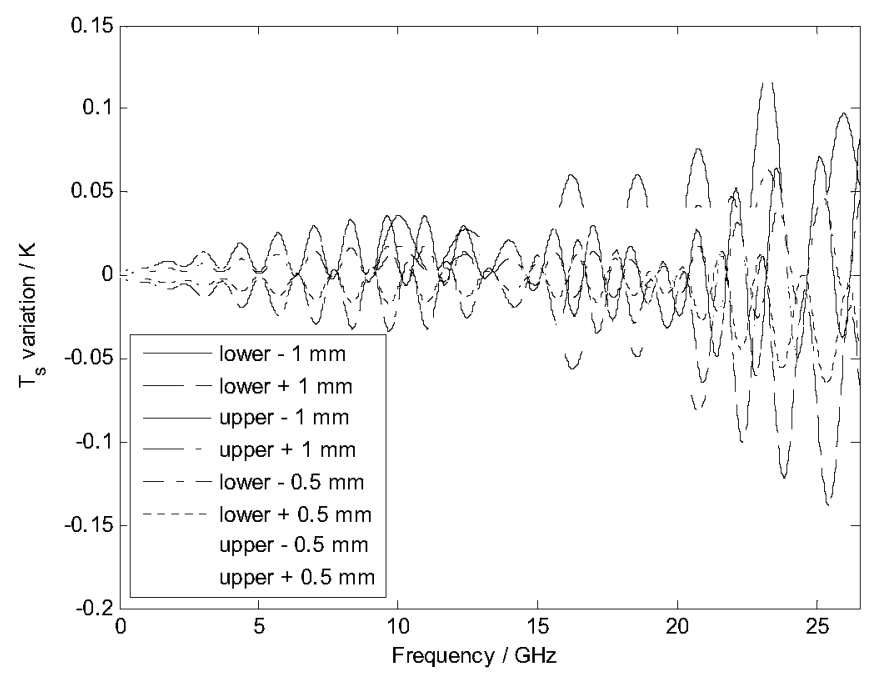

Figure 16. Noise temperature variation with the uncertainty in the level of liquid nitrogen.

are depicted in figure 6 . A separate case study for diameters and lengths has been performed for $\pm 0.01 \mathrm{~mm}$ tolerances. The variation in noise temperature for different combinations of tolerances is shown in figure 17. This small tolerance produces appreciable variations. Therefore, a high-quality manufacturing is needed for the thermal bead part. The manufacturing quality is also critical for the orifices, as shown in figure 18.

The roughness of the conductor surfaces increases the effective area and, consequently, the losses. We follow here the approach in [6] and express this uncertainty as an increase in the attenuation constant. Different increment percentages of $\alpha_{c}$ yield the noise temperature variation shown in figure 19. In [6] a $2 \%$ increase is assumed, which in this case yields variations between $0.047 \mathrm{~K}$ and $0.072 \mathrm{~K}$, depending on the nitrogen level, for the highest frequency.

\subsection{Specifications for materials}

The values for the dielectric constant and for the loss tangent of the boron nitride grade AX05 used in this work are those provided by the manufacturer. A rigorous measurement based on a perturbation technique in a resonant cavity must be performed in order to determine the uncertainty in these parameters and also the variation with the frequency. Figure 20 shows the variation in the noise temperature for an uncertainty of $\pm 5 \%$ in both parameters. In this case, a greater variation associated with the uncertainty in the loss tangent can be observed, since the loss tangent directly affects losses and, consequently, the thermal noise. It is important to note here that lower losses would lead to smaller variations in the noise temperature, but, on the other hand, the measurement uncertainty of very small loss tangent increases, with uncertainties around $100 \%$ for values between $10^{-5}$ and $10^{-4}$

The electrical conductivity of the conductor materials, which is needed for a wide range of temperatures ( $76 \mathrm{~K}$ to $297 \mathrm{~K}$ ), has been obtained from $[9,10]$. An uncertainty of $\pm 5 \%$ produces variations below $\pm 0.15 \mathrm{~K}$ for high frequencies, as 

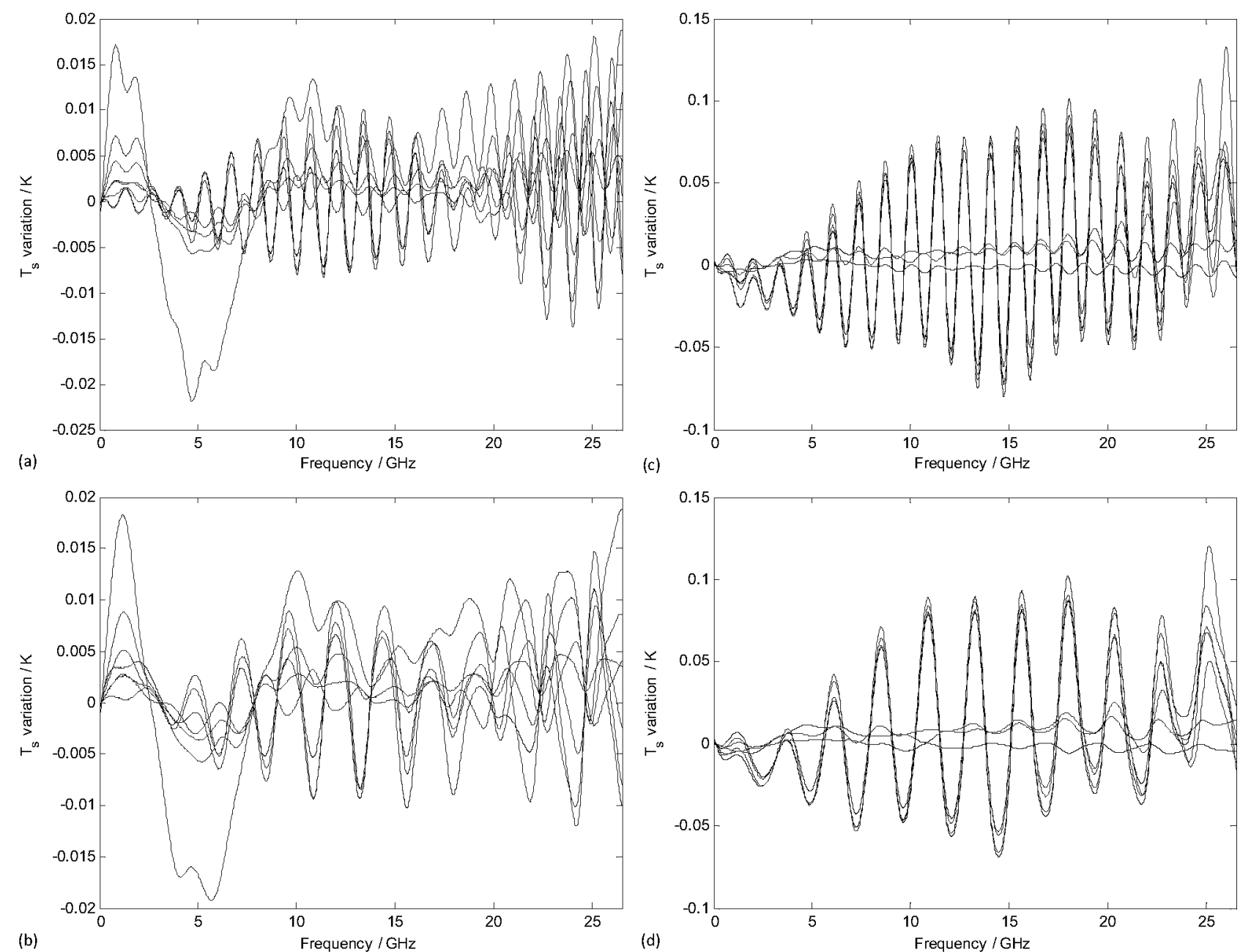

Figure 17. Noise temperature variation with the tolerance $(10 \mu \mathrm{m})$ in the manufacturing of the thermal bead and inner conductor modification: (a) length, lower level, (b) length, upper level, (c) diameter, lower level, $(d)$ diameter, upper level.

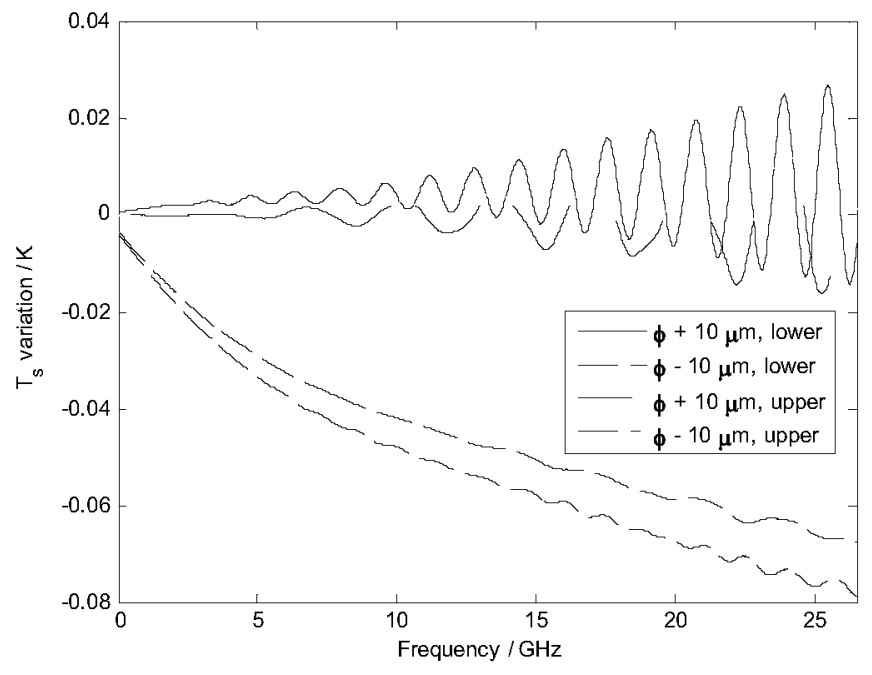

Figure 18. Noise temperature variation with the tolerance $(10 \mu \mathrm{m})$ in the manufacturing of the lower and upper orifices.

shown in figure 21. Therefore, accurate specifications for the electrical conductivity of the conductors are of key importance in order to reduce the uncertainty of the noise standard. The electrical conductivity can vary even with the manufacturing

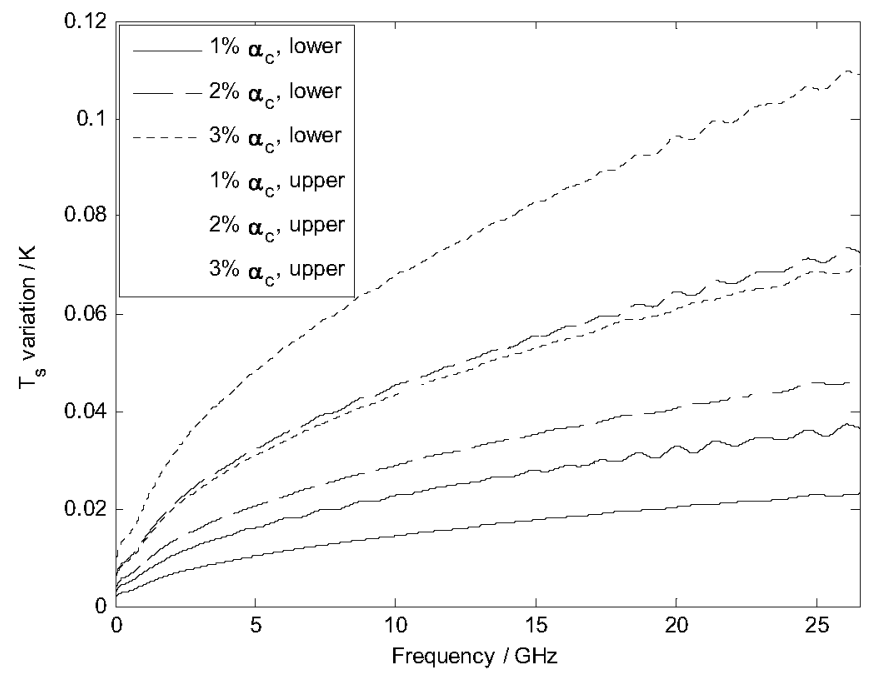

Figure 19. Noise temperature variation with the conductor roughness.

process within a specific conductor alloy, and the manufacturer does not provide accurate specifications for a wide range of temperatures. Therefore, a measurement of this parameter is strongly recommended. 


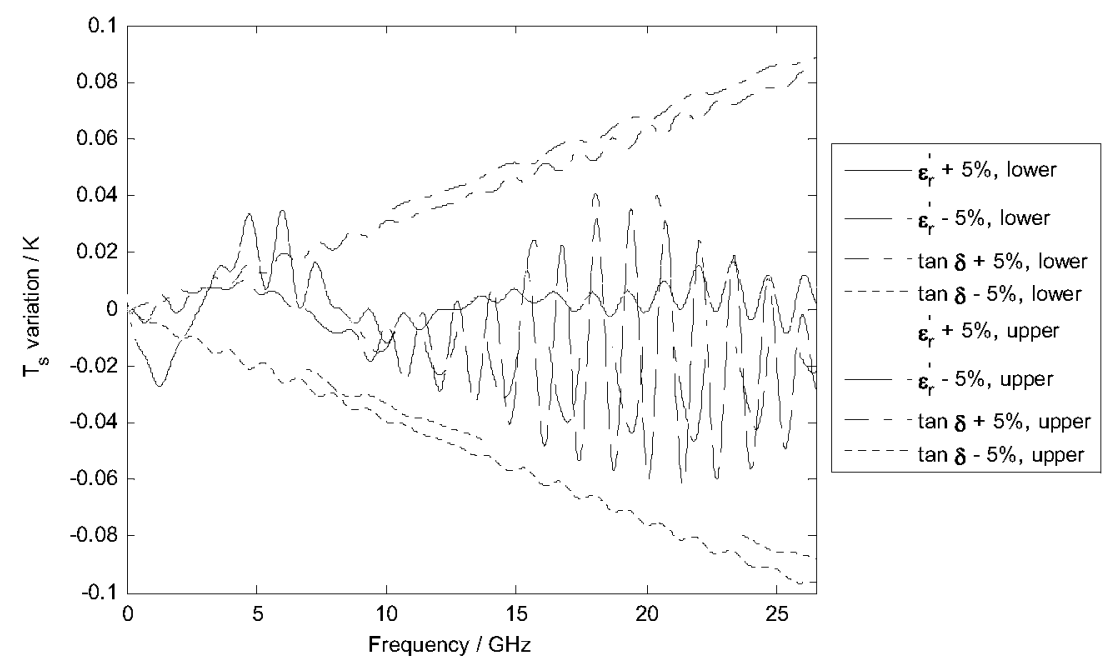

Figure 20. Noise temperature variation with the uncertainty in the boron nitride permittivity.

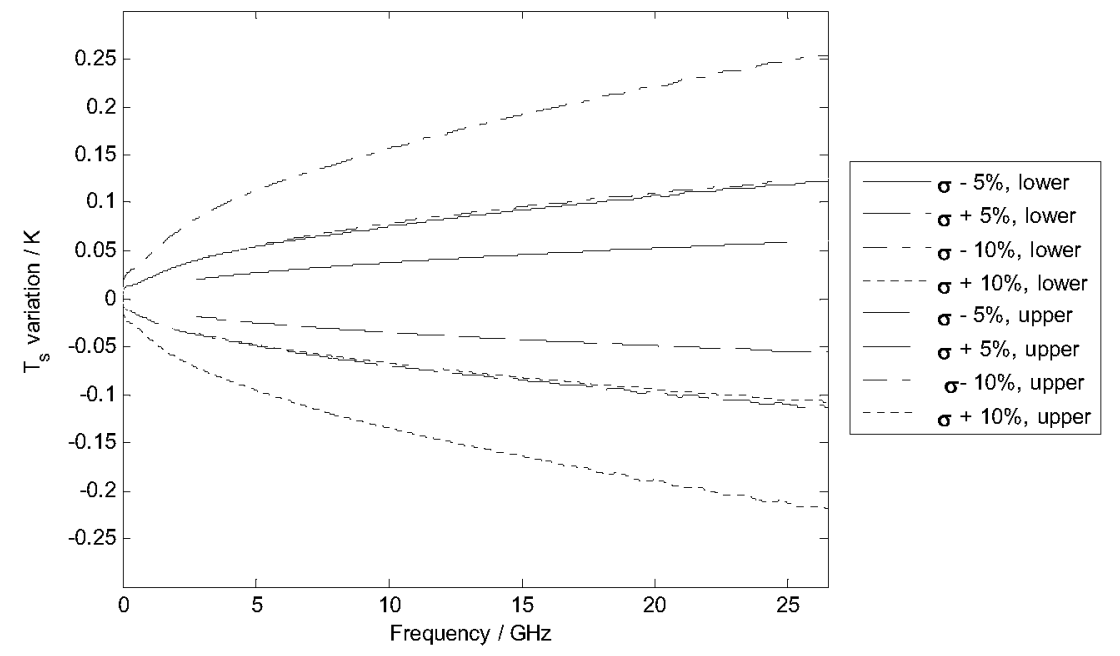

Figure 21. Noise temperature variation with the uncertainty in the electrical conductivity data for $\mathrm{Be}-\mathrm{Cu}$ and gold.

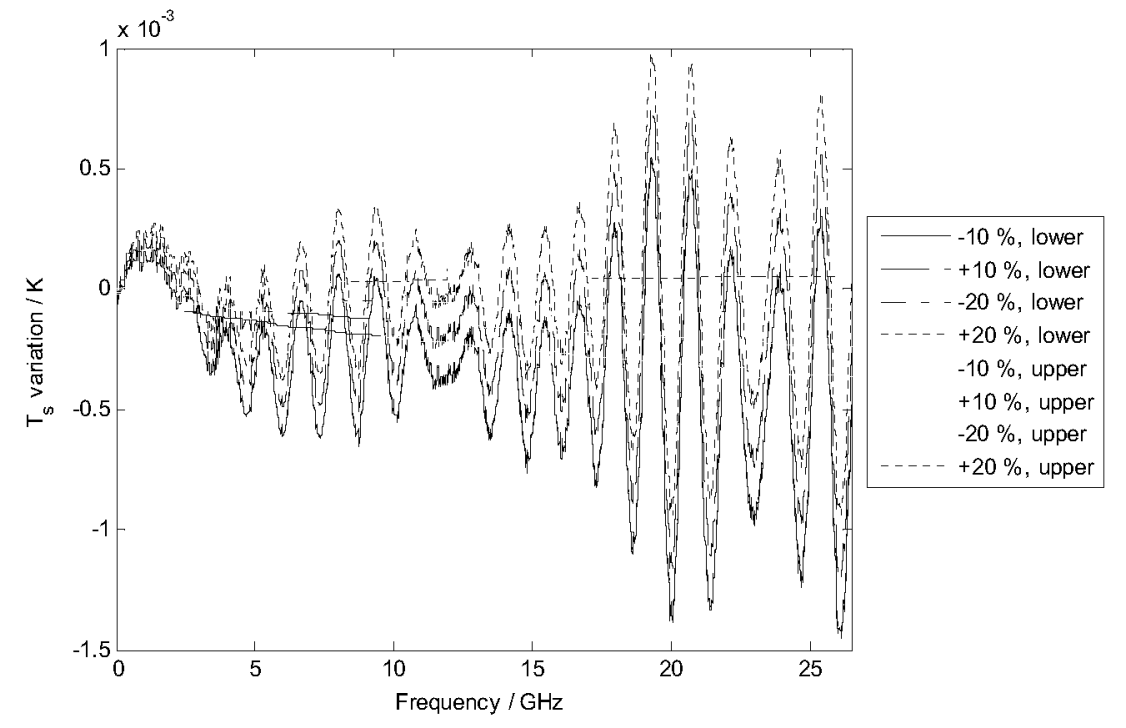

Figure 22. Noise temperature variation with the uncertainty in the thermal conductivity for $\mathrm{Be}-\mathrm{Cu}$ and boron nitride. 


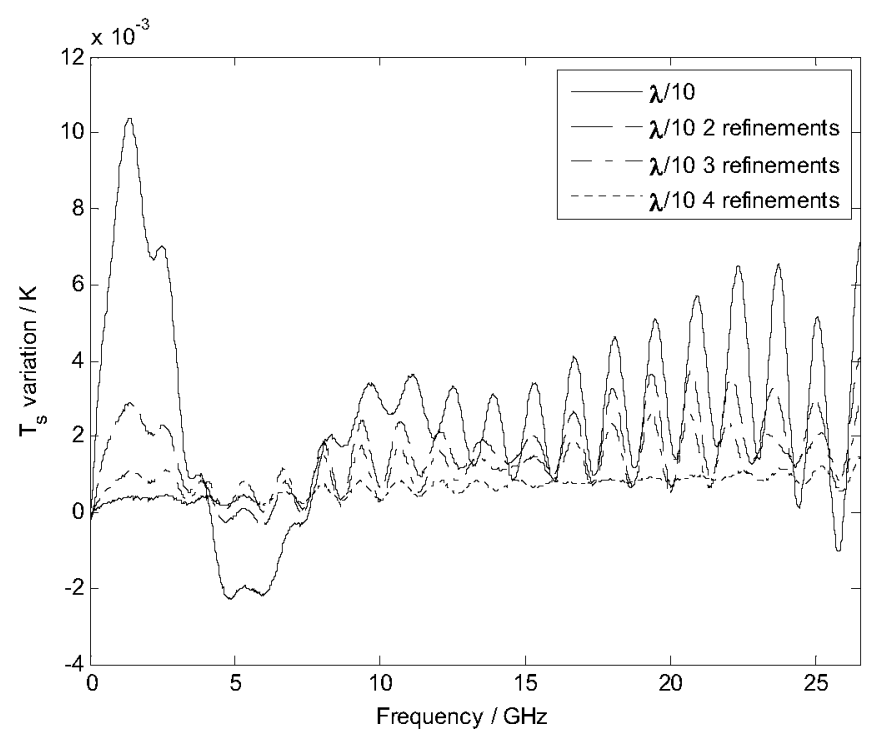

Figure 23. Noise temperature variation associated with the error in the numerical electromagnetic simulation of the thermal bead and orifices.

The thermal conductivity, specific heat and density of the beryllium-copper have been obtained from $[10,16]$. Thermal properties of gold are not needed in this approach since its small thickness $(0.75 \mu \mathrm{m})$ makes its thermal effect negligible. Boron nitride thermal properties are obtained from the manufacturer. With these data, the thermal simulations indicate that the thermal equilibrium is reached in less than 10 min. Temperature distribution at the thermal equilibrium does not depend on the specific heat or density of the materials, so it is not necessary to perform a sensitivity analysis for these two parameters.

A variation of $\pm 20 \%$ in the thermal conductivity generates variations $< \pm 0.0015$ in the noise temperature, as shown in figure 22 , which can be considered negligible. It is evident that the noise temperature sensitivity turns out to be greater with respect to the electrical properties than with respect to the thermal properties.

\subsection{Numerical simulations and integration}

In the noise temperature calculation, three different numerical computations are needed: (i) the thermal simulation for the determination of the temperature distribution throughout the standard; (ii) the electromagnetic simulation for the determination of the scattering parameters of the orifices and the thermal bead and (iii) the integration of (4). These operations increase the total noise temperature uncertainty because of their implicit numerical errors. A case study of these errors is necessary in order to minimize their effect.

It is obvious that, when a numerical method is required, the exact solution of a problem is unknown. Therefore, an estimation of the error in a numerical simulation is usually obtained by assuming that the exact solution is obtained by means of a much greater number of unknowns. This is the approach used here. Thus, for the numerical simulation of temperature distribution, meshes of 4150 (upper level)

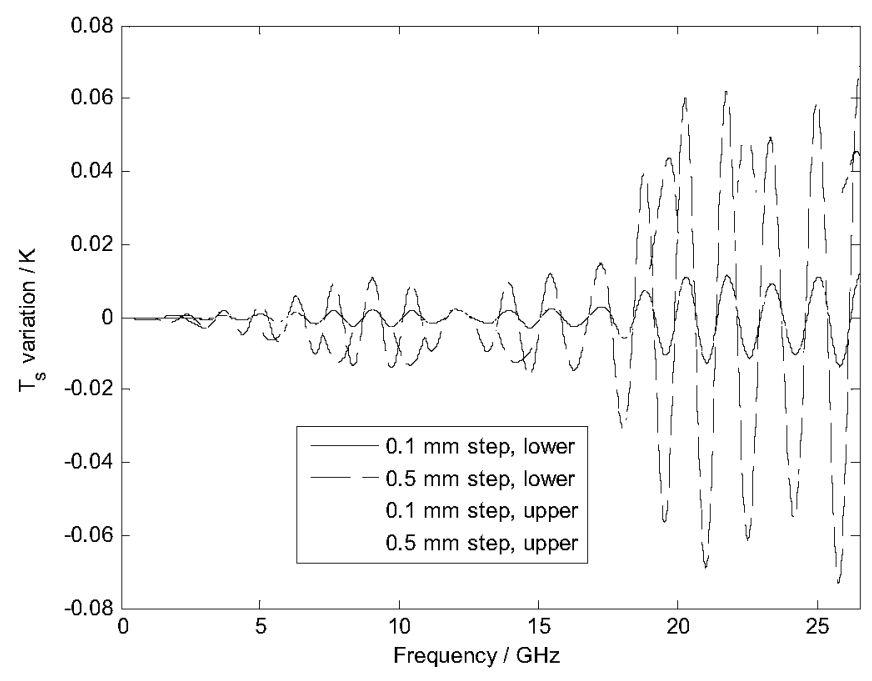

Figure 24. Noise temperature variation associated with the error in the numerical integration of (4).

and 7308 (lower level) unknowns are used. Comparing these results with those calculated with the reference meshes obtained by an extra refinement, variations in the noise temperature smaller than $\pm 0.006 \mathrm{~K}$ are obtained.

The finite difference time domain (FDTD) and FEFD methods have been tested for the electromagnetic simulation of the orifice and thermal bead. The latter was chosen since it showed a more stable convergence of the solution when increasing the number of unknowns. Figure 23 shows the variation in the noise temperature for different mesh densities. The reference mesh density for the estimation of the 'exact' solution was obtained with an element size of $\lambda / 10$ and five adaptive refinements. These results show that, in order to consider the error as negligible, the adaptive finite element method needs at least two refinement steps.

The trapezoidal rule has been used for the numerical integration in (4), which must be performed in segments 4-5, 6-8 and $8-l$. Figure 24 shows the effect of the integration step size. A reference 'exact' solution for a $0.01 \mathrm{~mm}$ step has been assumed. From these results, a $0.1 \mathrm{~mm}$ step has been finally chosen as a trade-off between accuracy and computation time.

Other uncertainty contributions such as those associated with the data of thermal conductivity, dielectric constant and loss tangent of liquid nitrogen, gaseous nitrogen and air are considered negligible. Although important for other standards, such as the impedance standard, the tolerances in the diameter of the inner conductor and in the inner diameter of the outer conductor of the coaxial line are in this case small enough to neglect their contribution to the overall uncertainty.

\section{Conclusions}

With the goal of developing a new primary noise standard that increases the frequency range of previous primary standards in coaxial technology, a computational tool for obtaining the noise temperature of the standard has been developed. The combination of analytical and numerical computations allows one to take into account a great number of parameters of the 
noise standard and to develop a sensitivity analysis in order to point out the characteristics of the noise standard that are critical for a low noise temperature uncertainty.

This analysis shows that the quality in the bead manufacturing, the accuracy in the electrical properties of conductors and the loss tangent in boron nitride, and the liquid nitrogen level are the main contributions to the noise temperature uncertainty. Therefore, a level control mechanism, an accurate permittivity and electrical conductivity measurements, and a high-quality manufacturing process are necessary to reduce this uncertainty. An important note here is that the sensitivity to the level of liquid nitrogen and to the manufacturing tolerances of the thermal bead strongly depends on the reflectivity of the noise standard. Therefore, the reduction in the internal reflections (for instance by new designs that eliminate one or both orifices, or by improving the bead design) would lead to reductions in these sensitivities.

Other results of this analysis allow one to fix tolerances, such as gold plating thickness or bead realization, in the manufacture of the standard, and also to assess the upper and lower bounds for the liquid nitrogen level.

It is important to note that uncertainty studies, such as the GUM methodology or even a Monte Carlo analysis, can also be performed by means of this computational scheme, prior to the manufacturing of the noise standard.

\section{Acknowledgments}

This work was supported by the Ministry of Science and Innovation (Spain), within the National Plan of Scientific Research, Development and Technological Innovation 20082011, under Project TRA2009-0281, and by INTA, Spain.

\section{References}

[1] Achkar J 1999 A set of waveguide primary thermal noise standards and related calibration systems for the frequency range 8.2-40 GHz IEEE Trans. Instrum. Meas. 48 638-41
[2] Buchholz F I and Kessel W 1993 A new primary thermal noise standard at PTB for the frequency range $12.4-18.0 \mathrm{GHz}$ IEEE Trans. Instrum. Meas. 42 258-63

[3] Buchholz F I, Kessel W and Niemeyer J 1989 Improvements in the accuracy of a new coaxial thermal noise standard IEEE Trans. Instrum. Meas. 38 465-9

[4] Randa J, Achkar J, Buchholz F I, Colard T, Rice J, Schubert D, Sinclair M and Williams G K 1999 International comparison of thermal noise-temperature measurements at 2, 4, and $12 \mathrm{GHz}$ IEEE Trans. Instrum. Meas. 48 174-7

[5] Kang T W, Kim J H, Yurchuk E F, Park J I, Sargsyan M V, Arsaev I E and Ouzdin R I 2007 Design, construction, and performance evaluation of a cryogenic 7-mm coaxial noise standard IEEE Trans. Instrum. Meas. 56 439-43

[6] Daywitt W C 1984 A coaxial noise standard for the $1 \mathrm{GHz}$ to $12.4 \mathrm{GHz}$ frequency range NBS Technical Note 1074 (NIST)

[7] HP 909D Coaxial Termination dc-26.5 GHz Technical Data, www.agilent.com/find/products

[8] Daywitt W C 1995 Attenuation in a plated coaxial transmission line Metrologia 31 349-55

[9] Lide D R (ed) 1996-1997 Handbook of Chemistry and Physics 75th edn (New York: CRC Press)

[10] Jensen J E, Tuttle W A, Stewart R B, Brechna H and Prodell A G 1980 Selected Cryogenic Data Notebook (Upton, NY: Brookhaven National Laboratory)

[11] Baker-Jarvis J, Janezic M D, Riddle B, Holloway C L, Paulter N G and Blendell J E 2001 Dielectric and conductor-loss characterization and measurements on electronic packaging materials NIST Technical Note 1520

[12] Tummala R R 1992 Multichip packaging: a tutorial Proc. IEEE 80 1924-41

[13] Miyashiro F, Iwase N, Tsuge K, Ueno F, Nakahashi M and Takahashi T 1990 High thermal conductivity aluminum nitride ceramic substrates and packages IEEE Trans. Compon. Hybrids Manuf. Technol. 13 313-9

[14] Fornet-Ruiz J, Lozano-Guerrero A, Monzó-Cabrera J and Díaz-Morcillo A 2011 New broadband thermal noise primary standard in coaxial technology Proc. Int. Microwave Symp. (Baltimore, MD)

[15] International Organization for Standardization 1993 Guide to the Expression of Uncertainty in Measurement (GUM) (Geneva: International Organization for Standardization)

[16] Cverna F 2002 ASM Ready Reference. Thermal Properties of Metals (Materials Park, $\mathrm{OH}$ : ASM International) 